Military Technical College Kobry El-Kobbah, Cairo, Egypt

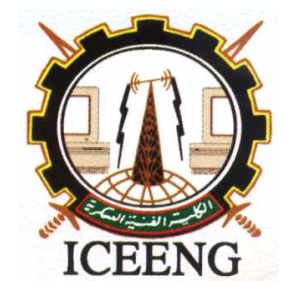

\author{
$6^{\text {th }}$ International Conference \\ on Electrical Engineering \\ ICEENG 2008
}

\title{
The low-latitude ionosphere: modelling of ionospheric effects on relative GPS measurements
}

By

Norsuzila Ya'acob *

Mardina Abdullah *

Mahamod Ismail *

\section{Abstract:}

Apart from geodesy and geophysical interest, GPS has great importance in scientific applications. For many years GPS has played a major role in determining ionospheric characteristics, including TEC and electron density profiles. The ionospheric effects in the propagation of Global Positioning System (GPS) signal is one of the main sources of error in GPS precise positioning. Therefore, it is important to have a precise estimation of the ionospheric delays. The developed model is a function of elevation angle and TEC. Then ionospheric correction model has been developed to accurately determine the difference in ionospheric delay so that a more accurate differential GPS correction can be made. A good model for the low-latitude region has become more and more important because of the need for high accuracy GPS positioning. An ionospheric error correction model should be made applicable to any location including the low-latitude region. The model has been validated and it gives the differential (between spaced receivers) ionospheric error to sub-centimetre accuracy.

\section{Keywords:}

Precise GPS positioning, ionosphere, differential GPS (dGPS), TEC

* Department of Electrical, Electronic and Systems Engineering, Universiti Kebangsaan Malaysia 


\section{Introduction:}

The earth's ionosphere which causes problems in many radio applications, especially in navigations, is now the subject of active research. The ionosphere is prone to significant disturbances, which are considerably worse during periods of high solar activity, such as at solar maximum [1]. The classical terrestrial techniques for positioning and navigation have dramatically changed in recent years in favour of the Global Positioning System (GPS). Application of GPS for ionospheric sensing is now the subject of worldwide interest. In addition to this application, it has also been used widely in ionospheric study to model the electron content whilst the GPS signals propagate through the ionosphere. The system, however, is mainly aimed at military [2, 3] purposes and with limited use for civilians.

A new approach in the determination of the differential (between spaced receivers) ionospheric error to sub-centimetre accuracy is described in this paper utilising a developed model. The developed model is a function of elevation angle and Total Electron Content (TEC). An ionospheric error correction model should be made applicable at any location including the equatorial region. Zain et al. [4] has reported on analysis of TEC using the GPS station at Arau, Perlis, in the northern part of Malaysia. Short term TEC analysis has also been done using GPS station at Miri, Sarawak [5]. While Ho. et al. [6] reported on the typical hourly variations for quiet ionosphere over Malaysia for 24 hours on July 14, 2000.

The aim of this work is to define and establish a mathematical model that represents the equatorial ionospheric conditions in order to provide capability to the single frequency GPS users to correct their observables from such effects. Data from Department of Geodesy, JUPEM (Jabatan Ukur dan Pemetaan Malaysia) provided the input to the model (Figure 1).

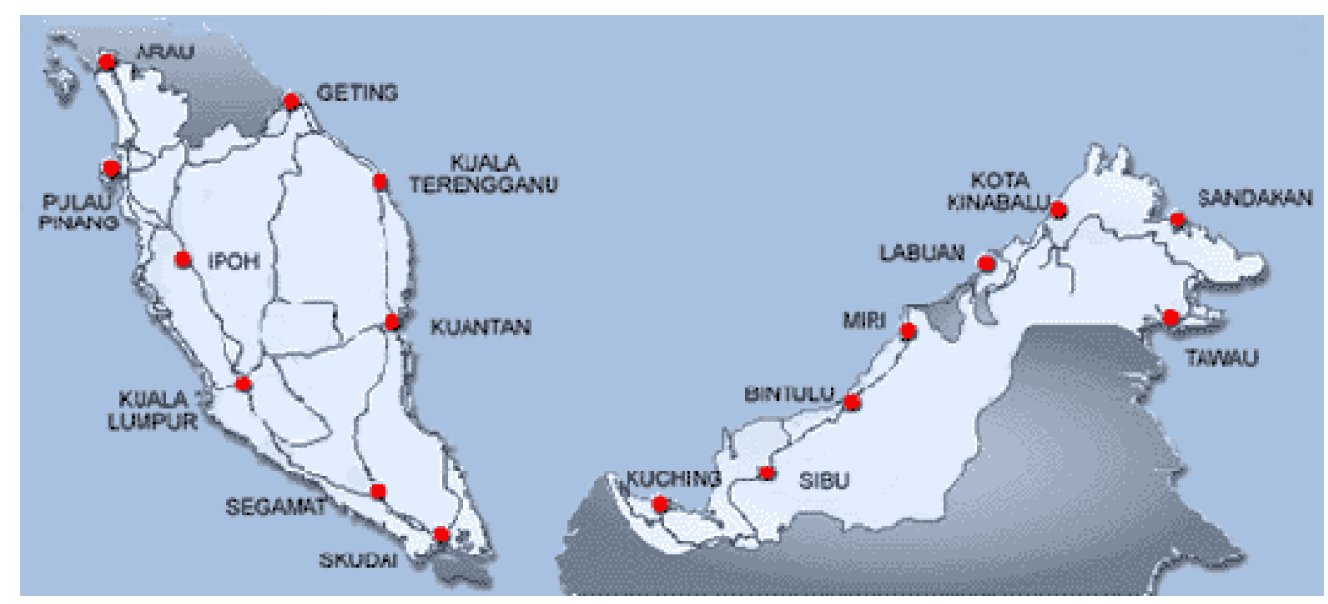

Figure (1): MASS Stations Sites [7] 


\section{GPS Relative Positioning:}

There are two basic methods for GPS positioning: absolute point positioning and relative positioning. In practice, code pseudo ranges are sufficient for most applications with sub-meter accuracy. Whereas, for sub-centimetre accuracy, the phase observable is used. For a better accuracy and minimized systematic errors, relative positioning techniques are used. This requires one fixed reference station with known coordinates (surveyed location) and another static or roving station. Both of these two stations receive the same GPS signal from the same satellite and have some common pseudo range error components, which can be removed. The reference station uses its known location to compute the error correction factor and transmits it to the static or roving receiver with unknown position to correct its measurements. By applying the corrections to the signal received, a user can typically improve the accuracy down to 2-5 m level or better [8]. This technique is called Differential GPS (dGPS) and is based on the double difference method.

\section{Ionosphere:}

The ionosphere is strongly related to solar activity that has an 11-year cycle. Geomagnetic activity shows a clear correlation with the sunspot cycle and has a peak occurring close to the sunspot maximum. More accurate values of the magnetic field can be obtained by using the International Geomagnetic Reference Fields (IGRF) [9]. The common and important index to describe the intensity of the field is called Kp index (p: planetary). This index is directly related to the three-hour index ap, and the daily indexes Ap, Cp and C9 [10]. Monthly published data can be obtained from SolarGeophysical Data report [11]. The parameter of the ionosphere that produces most of the effects on GPS signal is the total number of electrons (TEC) along the path.

\subsection{Ionosphere Model:}

Ionospheric models are usually computed by determining the TEC in the direction of all GPS satellites in view from a ground GPS network. The simple but less accurate method is the Klobuchar model for single frequency where the required parameters are available from the ephemeries data [12]. The short baseline distance of about $20 \mathrm{~km}$ was processed from Wisma Tanah Kuala Lumpur, KTPK $\left(3^{\circ} 10^{\prime} 15.44\right.$ 'N; $101^{\circ} 43^{\prime}$ $\left.03.35^{\prime \prime} \mathrm{E}\right)$ as a reference station and UPM Serdang, UPMS $\left(2^{\circ} 59^{\prime} 36.22^{\prime \prime} \mathrm{N} ; 101^{\circ} 43^{\prime}\right.$ $\left.24.63^{\prime \prime} \mathrm{E}\right)$ as a mobile station. The difference in ionospheric induced error between two stations can be expanded as: 


$$
\begin{aligned}
\frac{\Delta t_{d}(\text { TEC })}{\Delta L O S} & =\frac{1}{f(\beta)} f(\text { TEC }) \\
\Delta t_{d}(\beta, T E C) & =\frac{\Delta L O S}{f(\beta)} f(\text { TEC }) \\
f(T E C) & =\frac{\text { TEC from satellite to receiver path }}{\text { TEC used in } f(\beta)}
\end{aligned}
$$

where

TEC : total electron content

$\triangle L O S$ : differential in line of sight

$\beta$ : elevation angle

$\Delta t d$ : differential delay, in meter

From this model [13], there are two main parameters that need to be determined: the difference in LOS and TEC. Apart from these, the ratio as a function of elevation angles was examined.

\subsubsection{Modelling the Ionosphere using 10 order Polynomial:}

The ratio that was modelled by a polynomial function as define in eqn. (2). The polynomial coefficient is almost constant for any baseline length and elevation angle.

$$
\begin{aligned}
f(\beta)= & 6 \times 10^{2} \beta^{10}-2.7 \times 103 \beta^{9}+3.6 \times 10^{3} \beta^{8}-1.9 \times 10^{2} \beta^{7}- \\
& 1.3 \times 10^{2} \beta^{6}-6.2 \times 10^{3} \beta^{5}+1.1 \times 10^{4} \beta^{4}-2.4 \times 10^{4} \beta^{3}+ \\
& 3.8 \times 10^{4} \beta^{2}+1.5 \times 10^{5} \beta+3.5 \times 10^{5}
\end{aligned}
$$

\subsubsection{Line of sight (LOS):}

In this work the IGS fixed positions were used as an initial input to the calculation of the satellite position using broadcast ephemeris data. To obtain the LOS, the receiver and satellite positions should be known, and there are several methods to obtain them. The real time satellite position is sufficient in this application and the precision of LOS is not so crucial compared to other parameters in the model. From this calculation the difference in LOS between the spaced stations was obtained as defined below:

$$
\Delta L O S=L O S r e f-L O S m
$$

where

$\operatorname{LOS}_{\text {ref }}$ : line of sight at reference station

$L O S m$ : line of sight at mobile station 


\subsubsection{TEC Estimation Using GPS Measurements:}

A dual-frequency GPS receiver measures pseudo ranges and carrier phases at L1/L2 and its observables are used to compute TEC. In this work the method that uses data from dual frequency especially the pseudo range filtered by the carrier phase will be focused. The TEC data derived from GPS pseudo range measurements have a large uncertainty because of the high noise level of the pseudo ranges. In contrast, the noise level of carrier phase measurements is significantly lower. To reduce the effect of pseudo range noise on TEC data, GPS pseudo range data can be smoothened by carrier phase measurements. The "phase levelling" technique is used to compute precise phasederived slant TEC for each tracked satellite at each observation epoch [14]. The absolute slant TEC from the reference station can be measured directly from the normal dual frequency method expressed in (4).

$$
T E C=\frac{1}{40.3}\left(\frac{f_{1}^{2} f_{2}^{2}}{f_{1}^{2}-f_{2}^{2}}\right)(P 2-P 1)
$$

where

$f_{1}$ : carrier signals at $\mathrm{L} 1,1575.42 \mathrm{MHz}$

$f_{2}$ : carrier signals at $\mathrm{L} 2,1227.60 \mathrm{MHz}$

$P 1$ : pseudo range at $\mathrm{L} 1$

$P 2$ : pseudo range at $\mathrm{L} 2$

As slant TEC is a quantity which is dependent on the geometric ray path through the ionosphere, it is desirable to calculate an equivalent vertical value of TEC which is independent of the elevation of the ray path. The vertical GPS TEC is generally the main interest in the ionospheric research. Therefore, the slant TEC obtained from GPS observables is converted to a vertical value using a suitable mapping function. However, it is the conversion that introduces the error. This conversion introduces a few errors in the middle latitude where electron density is small. But it may result in obvious error at low latitude with large electron density and great gradient [15]. In order to refer the vertical TEC, the single layer thin-shell model was employed. In the analysis, the height of the single layer was set to $350-400 \mathrm{~km}$ above the earth's surface where the TEC vertical was determined. The mapping function is elevation dependent function which represents the ratio between vertical and slant TEC, given by the following formula.

$$
\operatorname{Sin} \chi^{\prime}=\frac{R_{E}}{R_{E}+h_{m}} \sin \chi
$$


where $R_{E}$ is the mean earth radius, $h_{m}$ is the height of maximum electron density, and $\chi$ and $\chi^{\prime}$ are the zenith angles at the receivers site and the ionospheric pierce point IPP, respectively.

\section{Result:}

GPS data from Wisma Tanah, Kuala Lumpur, KTPK as a reference station and UPM Serdang, UPMS as a mobile station on 8 November 2005 was collected, processed and analysed. The baseline length was about $20 \mathrm{~km}$. The GPS data was recorded in universal time system, whereby the sampling interval was $15 \mathrm{~s}$ and the cut-off elevation mask was $10^{\circ}$.

Figures 2 to 8 show representative cases of the different situations found in the analysis. The absolute slant TEC from the reference station can be measured directly by using the normal dual frequency method. The elevation angle for reference stations is $74^{\circ}$ for GPS satellite PRN 3. This can be calculated by using pseudo range and carrier phase measurements from the satellite. A model for computing the effects of the ionosphere was presented. The differential delay model can be seen for 1 hour (UT) and 3 hours (UT) for GPS satellites PRN 3 and PRN 23 respectively. The accuracy was achieved at $\mathrm{cm}$ level.

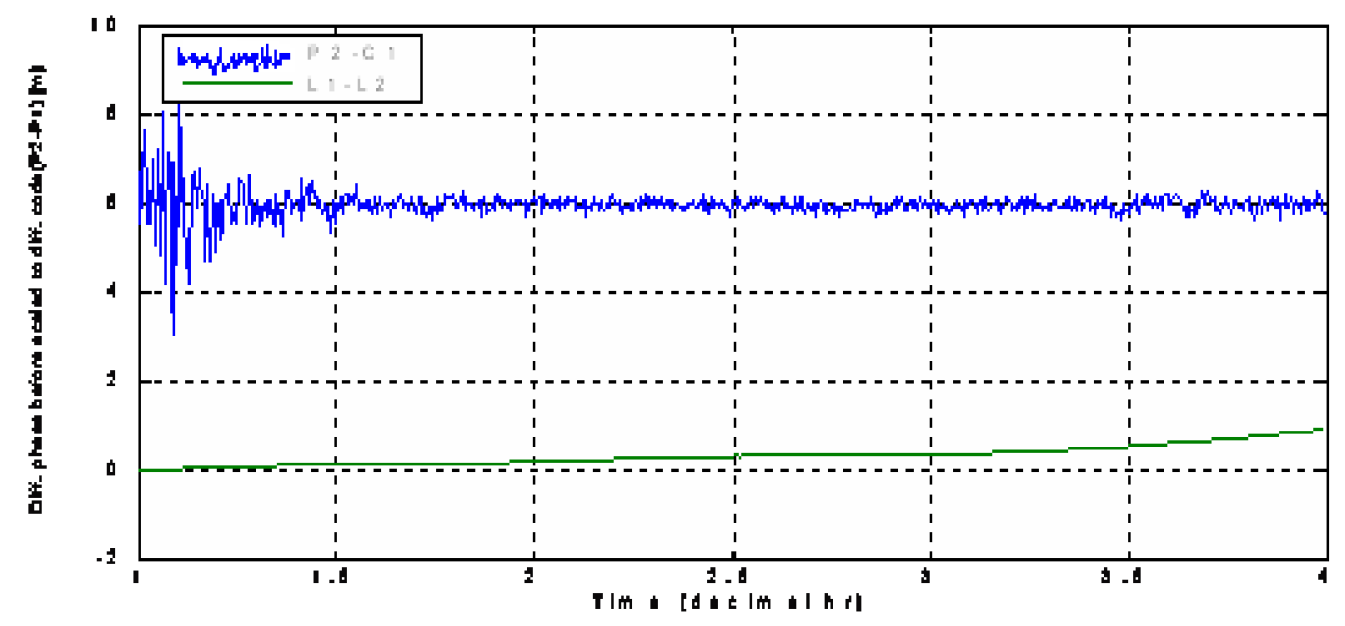

Figure (2):Different phase before scaled to different code (3 hour)for PRN 3

The plots in Figure 2 clearly indicate different phase before scaled to different code. The differential delay that can be calculated from P2-C1 of code measurements is noisy and influenced by multipath. Due to the ambiguity of the phase measurements the phase derived slant delay was scaled to zero relative range error at the first epoch. This eliminates the integer ambiguity provided there are no cycle slips. 


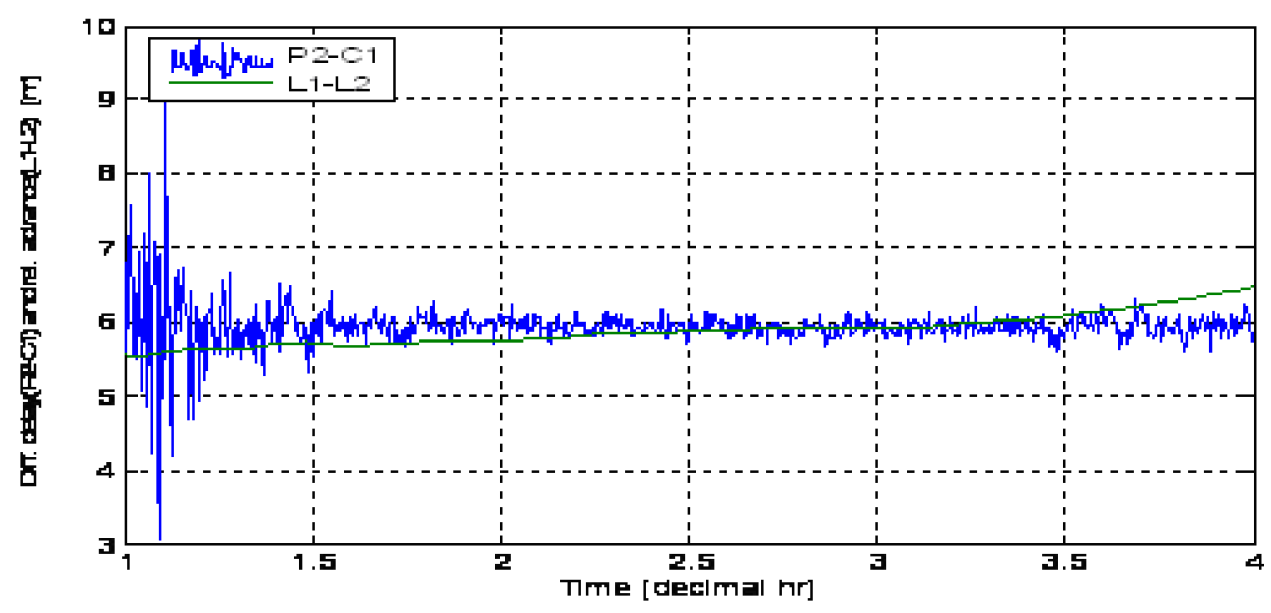

Figure (3): Different delay (P2-C1) and relative advance (L1-L2)for PRN 3

Figure 3 shows the differential delay (P2-C1) relative to phase advance (L1-L2). To eliminate the multipath effect code that is normally seen at both ends of the path or at low elevation angles, the differential delay code was fitted at the higher elevation angles. It must be noted that due to the multipath influence in the dual-frequency measurement, the higher elevation satellites have been chosen. This was done by defining a shift value and adding it to the relative phase to fit the code differential delay as can be seen in Figure 3. This is called "Levelling Process", which was applied to reduce multipath of the data and from Figure 4 is TEC slant scaled to (P2-C1) which is in TECU unit.

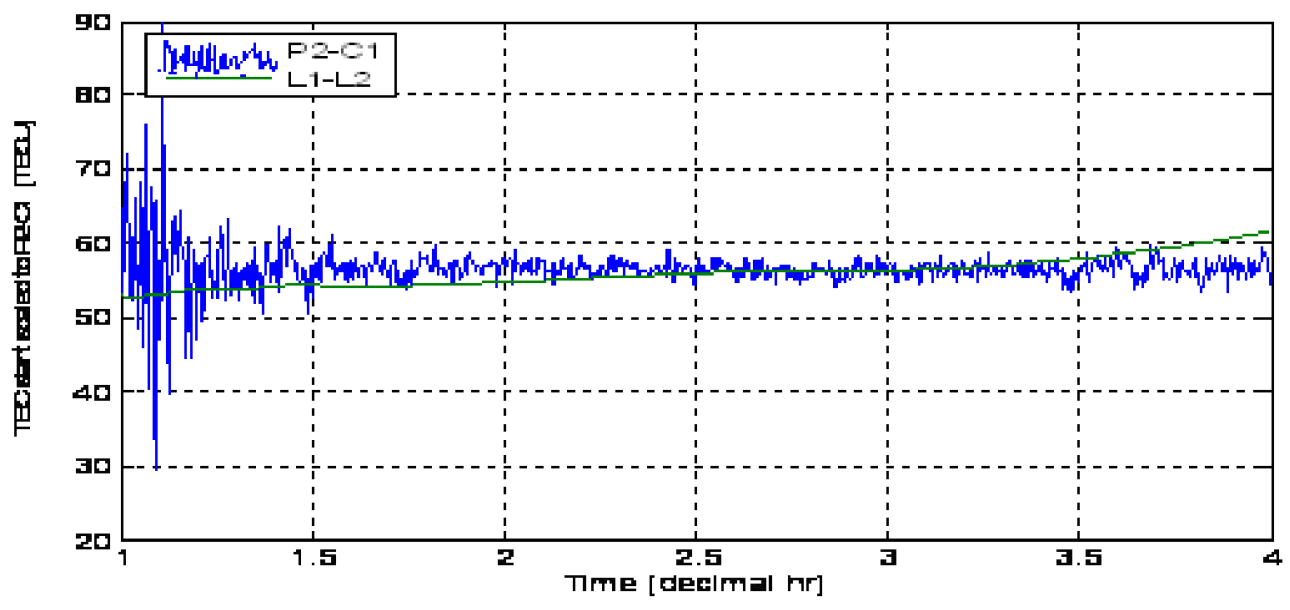

Figure (4): TEC Slant Scaled to (P2-C1) for PRN 3 


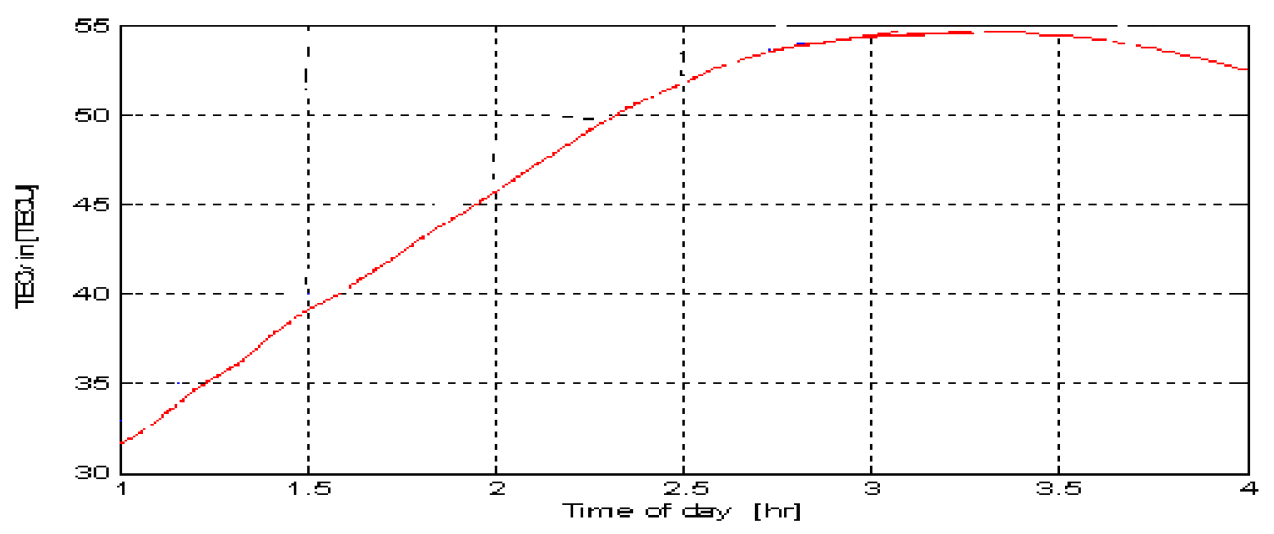

Figure (5): TEC vertical for PRN 3

Figure 5 shows TEC vertical for GPS satellite PRN3. Single Layer Model (SLM) was used to convert the slant TEC to vertical. This analysis at an equatorial region used SLM mapping function used. The peak altitude ranges from 350 to $500 \mathrm{~km}$ at equatorial latitudes. The final TEC values are precise, accurate and without multipath.

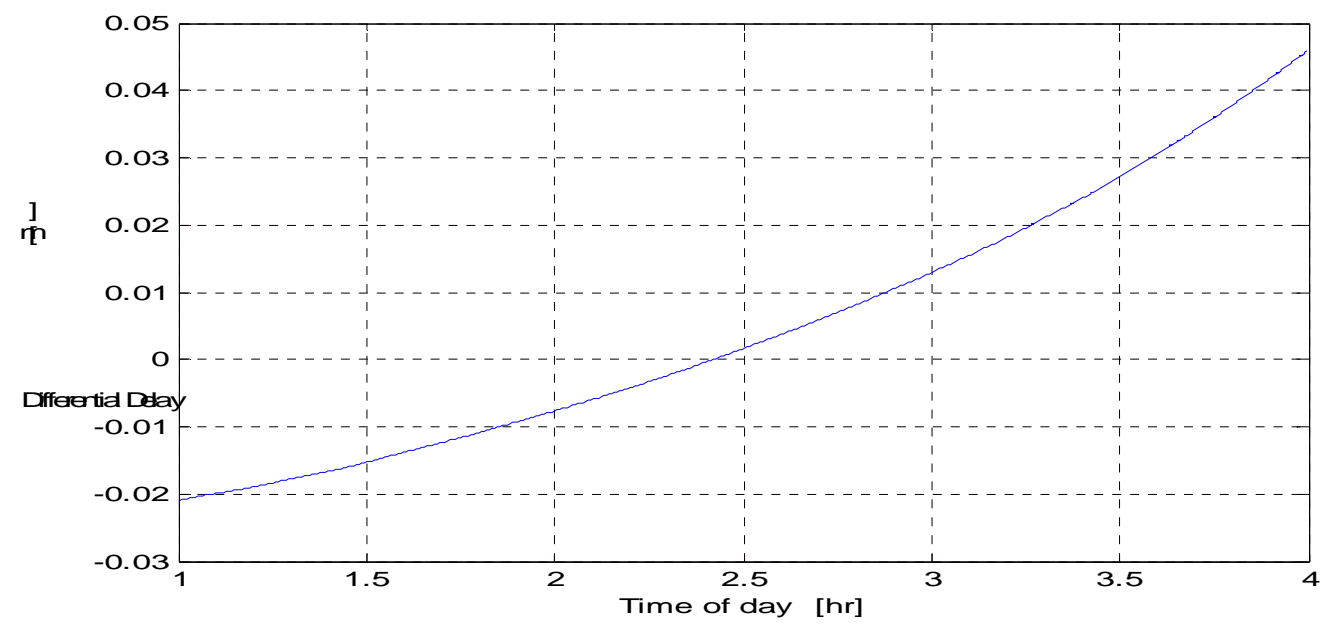

Figure (6): Differential Delay Model(3 hour) for PRN 3

The differential ionospheric delay $\left(\Delta \mathrm{t}_{\mathrm{d}}\right)$ calculated from the model is shown in Figure 6, 7 and 8. The correction model in (1) was used by substituting the above parameters at elevation angles roughly between $10^{\circ}$ and up to $90^{\circ}$, that were visible to the satellites for one hour with 15 second intervals. The accuracy was achieved at $\mathrm{cm}$ level. 


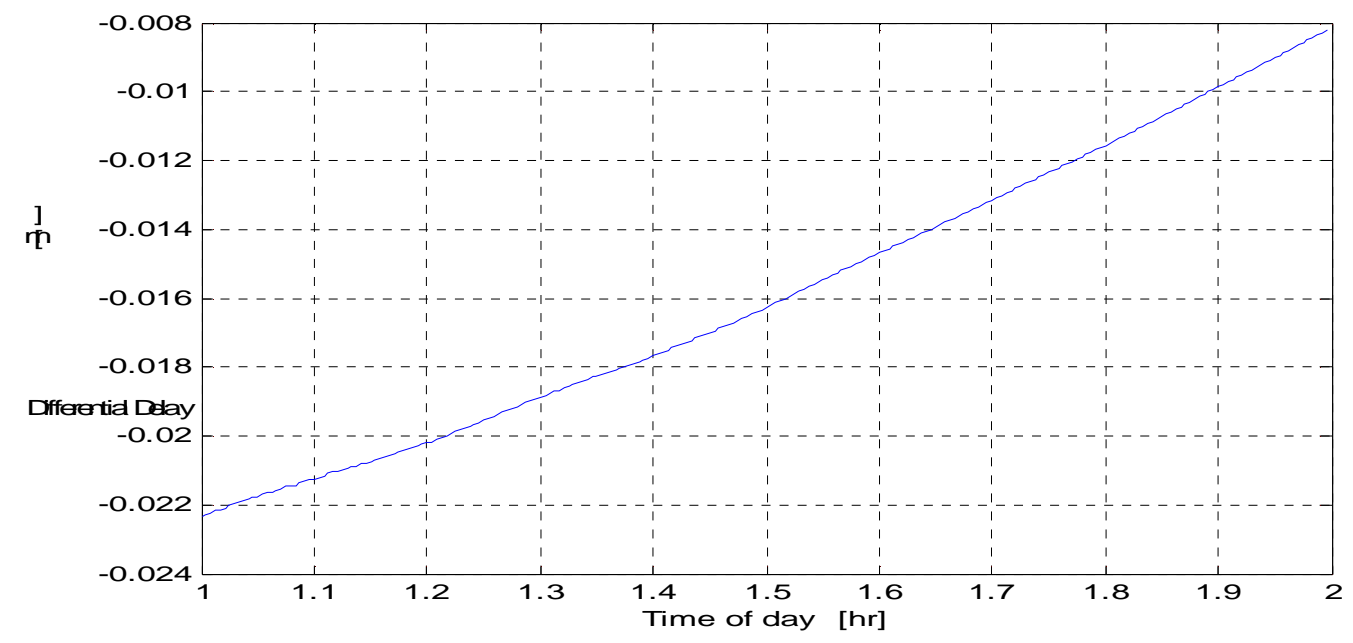

Figure (7): Differential Delay Model(1 hour)for PRN 3

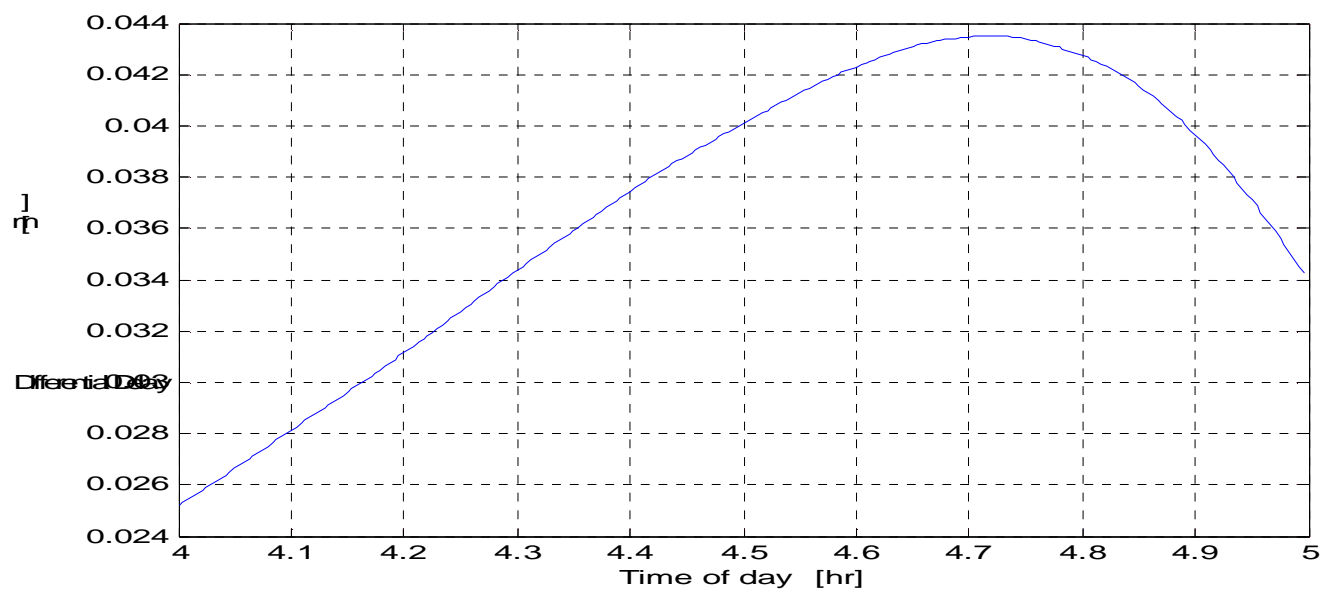

Figure (8): Differential Delay Model(1 hour)for PRN 23 


\section{Conclusion:}

Based on the experimental results obtained, the following conclusions can be drawn. The work presented here has shown promising results based on the utilisation of carrier phase observation for precise positioning. The developed algorithm is a function of elevation angle and TEC along the path to the satellite from the reference station, and is applicable to any location and TEC. From this model it is revealed that the required difference in line of sight ranges of satellite to receiver between both stations as its input parameter can be obtained from the ephemeris message. Furthermore, a cheaper single frequency receiver can utilise this model without suffering from higher noise when using an expensive geodetic frequency receiver.

The TEC used in this model only takes into account its time variation over the reference station and assumes that the ionospheric error are correlated over the mobile station. So this model is mostly suitable for short baseline. From the model we can get differential ionospheric delay in sub-centimetre accuracy.

\section{Acknowledgment:}

Jabatan Ukur dan Pemetaan Malaysia (JUPEM) has provided the GPS data in this paper. This work was partially funded by Science Fund (04-01-02-SF0191).

\section{References:}

[1] A. Dodson,T. Moore, Aquino, M.H. and S.Waugh, Ionospheric Scintillation Monitoring in Northern Europe, ION GPS 2001 Conference, Salt Lake City, Utah, USA, 2001.

[2] D.S. Coco, C. Coker, S.R. Dahlke and J.R. Clynch, Variability of GPS satellite Differential Group Delay Biases, IEEE Transaction on Aerospace and Electrical Systems, Vol 27, No 6, P 931-938.1991.

[3] J.M. Hasik and M. R. Rip, GPS At War: A Ten-Year Retrospective, in ION GPS 2001, Salt Lake City, UT,USA, The Institute of Navigation, P. 2406-17, 2001. 
[4] Zain A.F. M., and M. Abdullah, Initial Results of Total Electron Content Measurements Over Arau, Malaysia, Proc. $4^{\text {th }}$ IEEE Malaysia International Conference on Communications Vol. 1, 440-443, 1999.

[5] Zain A.F. M., and M. Abdullah, Measurements of Total Electron Content Variability at Miri, Sarawak: Short Term Analysis, Proc. $2^{\text {nd }}$ ICAST, Vol. 2., pages 1967 - 1775, Putrajaya, Malaysia, 15-17 August, 2000.

[6] Ho, Y. H., A.F.M. Zain and M. Abdullah, Hourly Variations Total Electron Content, TEC, For Quiet Ionosphere Over Malaysia, Proceeding of the Annual Workshop National Science Fellowship (NSF) 2001, pages 77-79, Petaling Jaya, 2002.

[7] Department of survey and mapping Malaysia, Malaysia Active GPS Malaysia. available: http://www.geodesi.jupem.gov.my/

M. Abdullah, H.J. Strangeways and D. M. A. Walsh, Accurate ionospheric error correction for differential GPS, in 12th International Conference on Antennas and Propagation, ICAP 2003, Exeter, UK, 2003.

[9] NASA, DGRF/IGRF geomagnetic field model 1945 - 2005 and related parameters. http://nssdc.gsfc.nasa.gov/space/model/models/igrf.html, 2004.

[10] G. Potsdam, Indices of Global Geomagnetic Activity. http://www.gfz potsdam.de/pb2/pb23/GeoMag/niemegk/kp index/, 2004.

[11] M. Abdullah, H.J. Strangeways and D. M. A. Walsh, Modelling and Determination of Ionospheric Effects on Relative GPS Measurements, $\mathrm{PhD}$ Thesis University of Leeds, 2004.

[12] Klobuchar, B. W. Parkinson \& J.J. Spilker, "Ionospheric effects on GPS, in Global Positioning System: theory and applications," 1st ed. American Institute of Aeronautics and Astronautics, Washington D.C., 1996. 
[13] M. Abdullah, H.J. Strangeways and D. M. A. Walsh, Accurate ionospheric error correction for differential GPS, in 12th International Conference on Antennas and Propagation, ICAP 2003, Exeter, UK, 2003.

[14] S. Schaer, Mapping and Predicting the Earth's Ionosphere Using The Global Positioning System, PhD Thesis, University of Bern, 1999.

[15] A. Komjathy, L. Sparks, A.J. Mannucci et.al., The ionospheric impact of the October 2003 storm events on wide area argumentation system. GPS solute. 9 (1), 41-50, 2005.

\section{Nomenclatures:}

TEC Total electron content

$\triangle$ LOS Differential in line of sight

$\beta$ Elevation angle

$\Delta$ td Differential delay, in meter

$\operatorname{LOS}_{\text {ref }}$ Line of sight at reference station

$\mathrm{LOS}_{\mathrm{m}}$ Line of sight at mobile station

$f_{1}$ Carrier signals at $\mathrm{L} 1,1575.42 \mathrm{MHz}$

$f_{2}$ Carrier signals at L2, $1227.60 \mathrm{MHz}$

P2 Pseudo range at L1

P2 Pseudo range at L2 